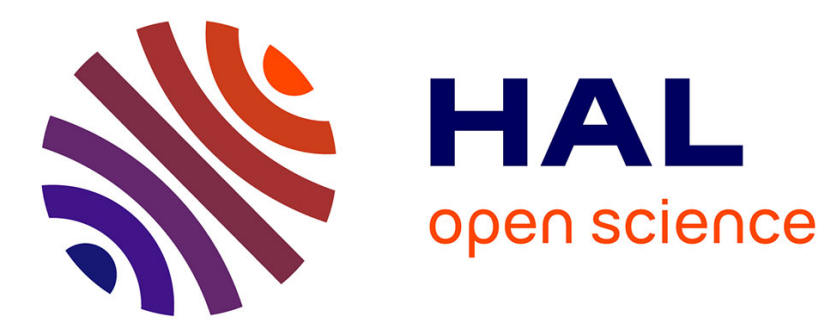

\title{
Sur un électromètre enregistreur à filament de carbone
}

P. Villard

\section{To cite this version:}

P. Villard. Sur un électromètre enregistreur à filament de carbone. Radium (Paris), 1911, 8 (12), pp.469-472. 10.1051/radium:01911008012046901 . jpa-00242518

\section{HAL Id: jpa-00242518 https://hal.science/jpa-00242518}

Submitted on 1 Jan 1911

HAL is a multi-disciplinary open access archive for the deposit and dissemination of scientific research documents, whether they are published or not. The documents may come from teaching and research institutions in France or abroad, or from public or private research centers.
L'archive ouverte pluridisciplinaire HAL, est destinée au dépôt et à la diffusion de documents scientifiques de niveau recherche, publiés ou non, émanant des établissements d'enseignement et de recherche français ou étrangers, des laboratoires publics ou privés. 


\title{
Sur un électromètre enregistreur à filament de carbone
}

\author{
Par P. VILlaRD
}

[École Normale Supćrieure. - Laboratoire de Chimic.]

Je me suis proposé de construire, en rue d'expériences sur l'électricité atmosphérique, un électromilre cnregistreur de firble volume, assez maniable pour pouvoir être installé dans un ballon sonde, et dont la sensibilité soit réglable à volonté, l'échelle entière comprenant, suivant les cas, plusieurs centaines de volts, ou seulement quelques volts.

L’in-trument, très habilement réalisé par M. Thur- 
neyssen, se présente extérieurement sous l'aspect d'unc boîte rectangulaire en laiton, munic des ouvertures nécessaires et fixée sur une planchette à la-

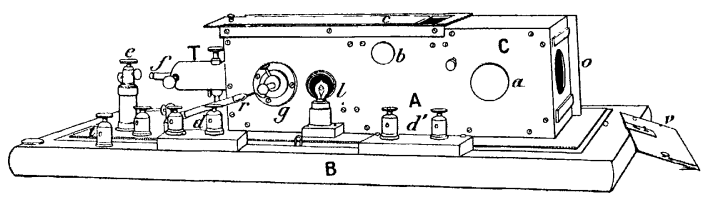

Fig. 1. - Aspect extéricur de l'électromètre.

$A$, boîte en laiton renfermant toutes les pièces de l'inctrument; $B$, planchette recevant lenveloppe protectrice en bois; (;, partıe contenant le châssis photographique; T, tule de laiton recevant la pièce qui porte le filament. On voit en $f$ l'extrémité de la tige à coulisse ì laquelle ce filament est fixé; $a$, vis de lixage du chàssis photographique ; $b$, bouton commandant la mise au point ; $c$, couvercle à colllisse; $d$, bornes reliées aux plateaux par l'intermé liaire des résistances liquides $r ; d^{\prime}$, bornes de li lampe $l ; e$, borne isolée à l’ambrotde. Un fil souple la met en communication avec $f ; t$, borne de terre; $y$, pièce isoléa recevant la tige qui porte l'un des plateaus; $o$, ouverture pour la mise au point, se fermant à l'aide du volet $v$; l, lampe.

quelle s'adapte une enveloppe protectrice en bois, analogue à celle des baromètres enregistreurs Richard (fig. 1 et 2 ).

Partie électrique. - L'électromètre proprement dit se compose de deux petits plateaux rectangulaires

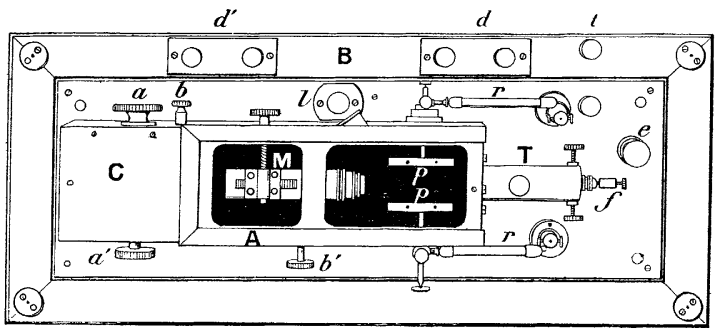

Fig. 2. - Projection horizontale de l'électromètre (couverele $c$ enlevé).

p. p., plateaux entre lesquels se déplace le filament; 11 , microscope; $a^{\prime}$. bouton molleté servant à la mise en place du chàssis enregistreur et à la mancuvre du cylindre; $b^{\prime}$, vis de décentrage.

Pour les autres lettres voir figure 1.

relićs aux deux pôles d'une pile sèche, et entre lesquels peut osciller librement un filament de lampe à incandescence, en forme d'U, tenu par une pince isolée à l'ambrö̈de, et qui constitue l'aiguille d'une sorte d'électromètre de Hankel.

Le filament de lampe, que j'ai déjà utilisé dans des électroscopes il y a une dizaine d'années, offre pour ce genre d'emploi des avantages considérables : le carbone qui le constitue est beaucoup plus léger que les métaux; parfaitement élastique et exempt de toute déformation résiduelle ou spontanée; le poids de l'aiguille et son moment d'inertie sont par suite très faibles, et le zéro est absolument stable. D'autre part, la forme en $\mathbf{U}$ supprime les déplacements autres que ceux perpendiculaires au plan des deux brins du fil.

Les filaments qui conviennent le mieux sont ceux des lampes de 110 volts 5 bougies ou 110 volts 10 bougies. Ces derniers, sensiblement plus longs, donnent une plus grande sensibilité, qu'on augmentc d'ailleurs beaucoup en amincissant le filament dans une flamme.

Pour donner les meilleurs résultats, cet amincissement doit être, non pas uniforme, mais progressif, croissant de la base à l'extrémitć libre du filament; pour une flexibilité donnée sous l'action des forces électriques, on réduit ainsi notablement le moment d'inertie et l'influence de la pesanteur.

Un fil de carbone ainsi traité peut devenir assez flexible pour déceler le cinquantième de volt et la résistance de l'air suffit à l'amortir complètement; toutefois, il est fragile et ne convient que pour une installation fixe ou l'emploi en ballon monté. Si l'appareil doit être confié à un cerf-volant ou à un ballon sonde, il convient de ne réduire que modérément la section du filament et l'on corrige alors le défaut de sensibilité et d'amortissement au moyen d'une petite bande d'aluminium battu, collée avec une trace de suif près de l'extrémité libre de l'U; l'amortissement est alors suffisant et l'on peut encore déceler le dixième de volt.

Pour faire varier la sensibilité dans les limites indiquées plus haut, on dispose des moyens suivants : amincissement plus ou moins grand du filament et emploi d'une bande d'aluminium plus ou moins large; modification de la distance des plateaux entre lesquels se déplace l'aiguille; enfin, choix d'une pile de charge de voltage convenable.

Dispositif optique de lecture et d'enregistrement. - Les déplacements de l'extrémité libre du filament ne dépassent pas en général $2 \mathrm{~mm}$ à droite ou à gauche du zéro. L'amplification el la projection de ces déplacements sur un verre dépoli ou une surface sensible sont réalisées de la manière suivante (fig. 5) :

L'extrémité mobile du filament porte, collé au sommet de la courbure par une trace de cire, un très petit miroir cylindrique constitué par un tube capillaire en verre très mince argenté intérieurement; ce miroir qui a environ $1 \mathrm{~mm}$ de longueur sur $\frac{1}{10}$ à $\frac{2}{10}$ de millimètre de diamètre, ne pèse que quelques centièmes de milligramme et est aisément porté par des filaments même fortement amincis. Une lampe de 2 volts, pourvue d'un condenseur et placée derric̀re une fente de $5 \mathrm{~mm}$ de largeur, éclaire ce miroir et $\mathrm{y}$ fait apparaître un trait lumineux extrêmement fin, qu'un objectif de 'microscope, muni d'une ou deux lentilles divergen!es amplificatrices, projette sur un verre douci ou sur la fente d'un cylindre enregistreur disposé dans la boitte de l'appareil. Pour assurer li permanence de la mise au point, l'axe de l'objectif est disposé dans le prolongement de l'axe de symétrie du filament; la longueur de ce filament étant inva- 
riable, il en est de même de la distance de son extrémité à la lentille frontale de l'objectif, et le réglage se maintient mème si l'instrument vient à être incliné dans un sens quelconque.

$\Lambda$ vec un grossissement de 15 , le spot ainsi obtenu est extrèmement fin et sa netteté, qui ne dépend que des

lindre est en place. Uis déclic effectue la mise en route au moment voulu et un butoir automatique arrête le cylindre quand il a effectué un tour complet. Ce dispositif, analogue au chàssis des appareils photographiques ordinaires, permet d'installer la pellicule on de la changer sans qu'on soit obligé de transporter l'électromètre dans la chambre noire; 'en outre, il protège la surface sensible contre la lumière diffusée par les lentilles du microscope ou celle qui pourrait pénétrer par les joints de l'appareil.

Sensibilité. - Si l'appareil doit être employé à poste fixe, le filament peut être pris suffisamment fin pour que le spot se déplace de $4 \mathrm{~mm}$ à $5 \mathrm{~mm}$ par rolt, les plateaux étant à une distance de quelques millimètres et maintenus à une différence de $\left(l^{\prime}\right.$, filament ; $m$, miroir (dont les dimensions ont été exagérées pour le rendre visible); $\mathrm{S}$,
d'éclairage ; L. lentille condensatrice ; $\sigma o^{\prime} o^{\prime \prime}$, objectif du nicroscope et lentille amplificatrice.

qualités optiques du microscope, est assez grande pour supporter un agrandissement ultérieur de 5 à 4 fois.

Diverses dispositions permettent de régler convenablement les positions de la lampe, du filament de carbone et du microscope. La lampe est fixée dans une monture à coulisse qui permet de régler sa hauteur. Le filament mobile esı porté par une lige coulissant dans un tube pourvu d'un collier de serrage et fixé dans un bouchon d'ambroïde muni d'une garniture métallique; c'est cet ensemble qu'on installe dans le tube $\mathrm{T}$ (fig. 1 et 2 ) où un système de quatre vis permet d'agir sur sa direction suivant deux plans perpendiculaires.

Le microscope est pourru d'une crémaillire pour la mise au point et d'une vis de décentrage servant ì régler la position du spot sur la pellicule scnsible.

Le cylindre enregistreur (fig. 4) est enfermé
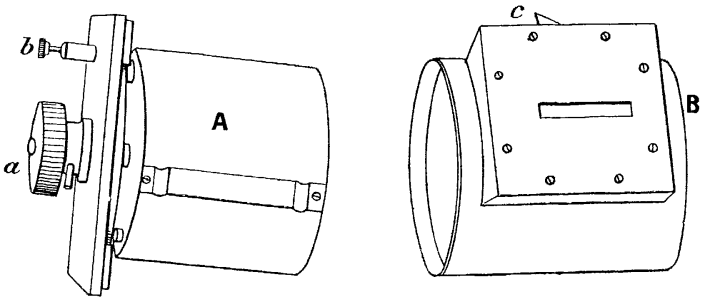

Fig. 4. - Chassis photoginphique ef eylindre enregistueur.

$\Lambda$, cylindre enregistreur ; $a$, bouton de mancruvre; $b$, verrou d'arrêt et de mise en marche; $B$, enveloppe métallique portant la fente, $c$, gâchette commandant l'ouverture de la fentr.

dans une enveloppe impénétrable à la lumière et munie d'une fente de $\frac{1}{20}$ de millimètre de largeur, qu'un volet démasque automatiquement quand le cy- potentiel de 100 à 120 volts .

Dans le cas ou l'électromètre doit être employé dans un ballon, il convient de prendre un filament plus solide et l'on n'obtient plus que $0,5 \mathrm{~mm}$ environ par volt, ce qui permet encore d'apprécier sans peine le dixième de volt.

Pour la mesure de fortes différences de potentiel (potenticl atmosphérique, par excmple), il est facile de réaliser, avec le même iustrument, une sensibilité d'un ordre entièrement différent. Il suffit de prendre un tilament plus court et d'écarter beaucoup les plateaux. I.'étendue de l'échelle peut alors atteindre 1000 ou 2000 volts.

Influence d'une inclinaison de l'instrument sur la position du zéro. La force antagoniste étant produite par l'élasticité du filament, l'appareil peut fonctionner dans n̈importe quelle position. Il est commode de le placer de telle sorte que les deux brins du filament soient horizontaux, le plan qui les contient étant vertical : les déviations se font alors dans un plan horizontal. Si l'électromètre doit être placé sur un support mobile tel que la nacelle d'un ballon, et exposé ainsi à de légers changements d'orientation, il convient de le disposer de telle sorte que les déviations se produisent dans un plan vertical. On voit aisément qu'une inclinaison, mème de quelques degrés, ne fait alors varier la position du zéro que d'une quantité tout à fait négligeable.

La figure 5 représente les courbes données directement par l'appareil. La première représente les variations de voltage d'une distribution ćlectrique urbaine, la seconde (sensibilité réduite) donne les variations du potentiel atmosphérique à quelques mètres du sol, pendant une durée de quelques heures. 
Rapidité des indications. - Par suitc de lit faible inertie el de l'amortissement nolable du filament, l'électromètre enregistre correctement des polentiels décclés el la sensibilité est nolablement diminuée. La vitesse de rotation dı tambour photographique et la puissance de la lampe doivent naturellement
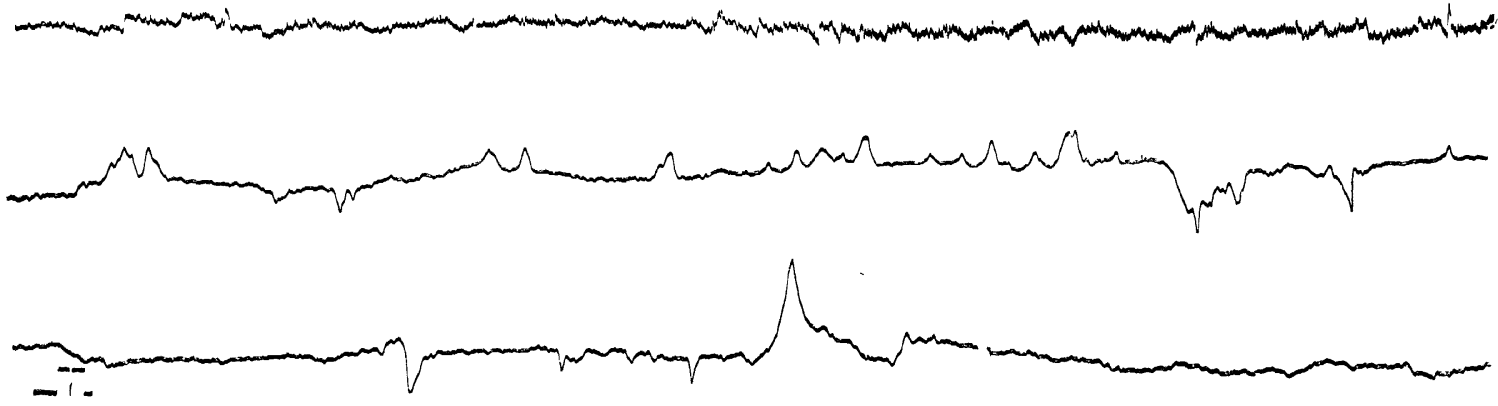

Fig. 5.

varianı avec la rapidité qui correspond à une fréquence de 5 à / par seconde. Le " pompage ) d'une dynamo entraînéc par une machine à vapeur (fréquence $\mathbf{2}$ environ) est par exemple mis en éridence d'une manière presque parfaite. Le filament mobile peut même suivre une différence de potentiel alternative de fréquence usuelle (40 à 50 par seconde) mais les harmoniques (fréquence 120 à 150,200 à 250 , etc.) ne sont pas être proportionnées à la rapidité de variation du potentiel qu'il s'agit d'enregistrer. Dans le cas du potentiel atmosphérique, il suffit que le film photographique se déplace à raison de $1 \mathrm{~mm}$ environ par minute et, dans ce cas, une lampe de 2 volls, 0,25 amp. donne un tracé d'une intensité tout à fait satisfaisante.

[Manuscrit reçu le 13 novembre 1911.] 